\title{
FILOSOFIA-SCIENZA E RELIGIONE, I NUOVI PERCORSI TRACCIATI DA HANS JONAS
}

Philosophy, Science and religion, new pathways traced by Hans Jonas

\begin{abstract}
Resumo: Il pensiero di Jonas, in particolare quello della sua seconda fase, dedicato alla filosofia della biologia, a pre nel confronto filosofico con la scienza nuovi spazi di riflessione critica per la filosofia e per le scienze stesse. In tale incontro entrambe si arricchiscono, acquisendo maggior coscienza metodologica e ampliando le prospettive di osservazione dei fenomeni. Sorge, così, in modo rinnovato, la possibilità di nuove soluzioni interpretative, che, ponendosi al di là di desueti schieramenti ideologici, riescano a comprendere più in profondità la complessità della realtà epocale a cui ci troviamo di fronte e dunque aumentino la capacità di governarla.
\end{abstract}

Parole Chiave: Filosofia, scienza, religione, Hans Jonas.

Abstract: Jonas' works, especially the ones dealing with the philosophy of biology, opened up new cri tic avenues in the philosophy and in the sciences. The result is an enrichment of both philosophy and science through an increased methodological awareness and a wider perspective. This leads to new solutions and interpretations that go beyond established frameworks and reach toward a deeper understanding of the complex reality in which we live, thus increasing our opportunities to govern its course.

Keyworks: Philosophy, science, religion, Hans Jonas.

\section{L'orizzonte della temporalità}

L'eterno non necessita di responsabilità, attende che vi si partecipi, tramite il desiderio che scaturisce dall'apparire mediato dal mondo dell'eterno bene.

La sete di eternità costituisce il senso dell' eros di origine platonica, mentre è «la sete di temporalità nelle sue concretizzazioni sempre nuove» il senso e la direzione 
della preoccupazione di Hans Jonas per la conservazione della specie umana. Ciò impone nuovi doveri epocali nell'era tecnologica che segue alla terza rivoluzione industriale, tra i quali però «non figura quello dell'aspirazione verso ciò che è perfetto e intrinsecamente definito» ${ }^{1}$.

Nelle pagine de II principio responsabilità ${ }^{2}$, intitolate L'orientamento "verticale" e non "orizzontale" dell'etica tradizionale, Jonas afferma che la filosofia di Platone, nel rappresentare una delle maggiori antitesi alla concezione moderna dell'essere e dell'etica, può ben essere una pietra di paragone per sottolineare la sua stessa posizione. L'eros platonico, infatti, non è responsabile per il suo oggetto eterno, che non "diventa", bensì "è"; il tempo non può nuocere a ciò cui non accade nulla. L'eros platonico, certo, è anche impulso affettivo rivolto al bene, dunque è fra tutte le pulsioni concorrenti nell'essere umano quella maggiormente determinata dalla causa materiale, senza fare di se stessa una virtù conseguente a una scelta autonoma e senza essere senso di responsabilità da parte del soggetto verso qualcosa che sia nel mondo o del mondo.

L'ontologia di Jonas non è un'ontologia di essenze immutabili, ma descrive l'essere in divenire del mondo dalle sue forme più semplici a quelle più complesse in una catena evolutivamente ascendente. Al suo apice, l'essere umano sviluppa una progressione continua dei propri poteri culturali, scientifico-tecnologici e, poiché possiede in modo precipuo la capacità di riflettere su se stesso e sul proprio operato, si trova sempre più in una situazione di responsabilità nei confronti dell'esistente discendente.

Hans Jonas procede con gli strumenti dell'analisi critica, della descrizione fenomenologica e della verifica/falsificazione della produzione teorica del soggetto nell'esperienza. Si confronta con le conoscenze raggiunte dalle scienze naturali e dalle scienze umane e giunge a una concezione ontologica che riesce a tenere in conto la

\footnotetext{
${ }^{1}$ H. JONAS, Das Prinzip Verantwortung. Versuch einer Ethik für die technologische Zivilisation, Frankfurt a/M, Insel Verlag, 1979, p. 227; trad. it. di P. Rinaudo, II principio responsabilità. Un'etica per la civiltà tecnologica, a cura e intr. di P.P. Portinaro, Torino, Einaudi, 1990, p. 157.

${ }^{2}$ Cfr. ivi, pp. 225-227; trad. it. cit., p. 155-157.
} 
materialità e l'energia che compongono i fenomeni del mondo. Anzi sostiene che proprio il sinolo di materialità ed energia dà luogo, in modo evidente a partire dal metabolismo degli organismi più elementari, al fenomeno della libertà. Nel bisogno costante di ricambio di materia dall'esterno l'organismo si trova in uno stato di trasformazione che lo emancipa sia dalla sua stessa sostanza sia dalla sostanza incamerata, rinnovandolo e rendendolo altro pur nella continuità della forma. Sintetizza in Organismo e libertà:

\begin{abstract}
La condizione originaria dell'organismo, persino a livello unicellulare, mostra l'individualità come un'avventura della libertà, con cui una forma mantiene la sua identi tà attraverso il cambiamento della sua materia. La libertà viene bilanciata dialetticamente dalla necessità, l'autonomia dalla dipendenza. L'ipseità qui adombrata ha sin dall'inizio il suo pendant nell'alterità del mondo ${ }^{3}$.
\end{abstract}

II progressivo accrescimento di tale dialettica con tutto il peso insito in esso è all'origine dello sviluppo della vita stessa dalle forme vegetali alle forme animali, fino all'uomo, che arrischiano maggiormente la loro esistenza in modo proporzionale all'aumento del movimento e dell'azione, all'aumento dell'autonomia e dello spazio di libertà conquistato.

Il fenomeno del vivente si è staccato con un atto di libertà dall'integrazione delle cose inanimate della materia e con questo atto di isolamento si è trovato in continuo bilico fra l'essere e il non essere, come possibilità propria del modo dell'essere vivente. Ogni organismo nel manifestare interesse alla continuazione ha acquisito e rafforzato un'identità che non può che costituirsi in una relazione dinamica di polarità quali la forma e la materia, il sé e il mondo, la libertà e la necessità, l'essere e il non essere.

\footnotetext{
${ }^{3}$ H. JONAS, Bewegung und Gefühl. Über die Tierseele, in ID., Organismus und Freiheit. Ansätze zu einer philosophischen Biologie, Göttingen, Vandenhoeck \& Ruprecht, 1973, p. 162; trad. it. di A. Patrucco Becchi, Movimento e sentimento. Sull'anima animale, in Organismo e libertà, a cura e intr. di P. Becchi, Torino, Einaudi, 1999, p. 147.
} 
Questa dimensione dinamica e relazionale è comune a ogni essente, a ogni sistema naturale e culturale, in quanto ciò che consente all'insieme di conservarsi, qualunque esso sia, è l'esistenza di un principio improvvisamente efficace, tramite il quale il molteplice riesce a organizzarsi e trovare un' unità, in un reciproco determinarsi delle parti. L'essere dell'essente, di una piccola come di una grande unità, è dovuto a un accadere relazionale che ha a che fare con la conservazione e la continua trasformazione dell'energia, con l'equilibrio e il disequilibrio delle parti, con i limiti dati dalla necessità della materia.

Nei sistemi vitali si assiste, inoltre, a fenomeni di causalità non lineare, di imprevedibilità e dunque di scelta e di libertà che li emancipano dialetticamente dalla necessità della materia ${ }^{4}$. Ciò è dovuto anche allo sviluppo di maggiori capacità di relazione che portano gli organismi al di là della propria originaria sostanza nella ricerca di supplire alla propria non-autosufficienza costitutiva.

L'essere, per Jonas, dunque è un accadere dinamico relazionale, fra la necessità della materia e l'indeterminatezza e la libertà dell'energia vitale, che si concretizza di volta in volta in sistemi costitutivamente aperti e transeunti. L'equilibrio momentaneo di tali concretizzazioni fra le parti e il tutto, che si attua nel tempo della loro esistenza, permette, comunque, l'individuazione di elementi di permanenza nel mutamento, di elementi meta-storici che insieme a quelli storici contribuiscono a rendere interpretabile il mondo nelle sue concretizzazioni di realtà divenienti che tendono all'autoconservazione.

II confronto con l'oggetto è per il soggetto umano la possibilità più propria dell'esperienza della verità mondana come falsificazione delle illusorie e fallaci interpretazioni e come ricerca iperbolica di adaequatio imaginis ad rem. Essendo in primis esercizio di negazione di ciò che non si ritiene vero, l'esperienza della verità è per l'essere umano prevalentemente vissuta nell'attività del pensiero critico che si

\footnotetext{
${ }^{4}$ Cfr. H. JONAS, Evolution and Freedom: On the Continuity among Life-Forms, in ID., Mortality and Morality. A Search for the Good after Auschwitz, L Voegel (ed., intr.), Evanston (Illinois), Northwestern University Press, 1996, pp. 59-74.
} 
attua in situazioni di libertà. L'esperienza della verità accade e si condivide là dove c'è libertà, dunque è testimonianza della possibilità della libertà, essendo la libertà prerequisito stesso della verità nella storia. Jonas in Organismo e libertà, afferma:

La condizioneinsita nell'uomo della possibilità della storia - la sua libertà, appunto - non è però essa stessa storica, bensì ontologica; e diviene essa stessa, se scoperta, il fatto centrale nell'evidenza da cui deve attingere ogni dottrina dell'essere ${ }^{5}$.

Egli, infatti, nella riflessione filosofica del secondo dopoguerra cerca di determinare ontologicamente l'essenza della libertà umana in relazione al restante mondo della vita, anzi all'intera natura. Individua proprio nell'interna trascendenza di quella libertà, pur nel rapporto dialetticamente necessario con i limiti della materia, l'indicazione per rintracciare metafisicamente un nuovo senso di trascendenza e perennità, in una prospettiva di partecipazione relazionale al divenire dell'essere che nel suo permanere dinamico rimanda a elementi metastorici.

La ricerca di ciò che è essenziale per l'essere umano non può non indagare il rapporto dell'uomo con l'essere che incontra negli enti naturali e culturali a lui circostanti, poiché quello è il luogo e il tempo entro i cui limiti ogni individuo realizza la sua esistenza e attua la sua libertà. L'identità dell'uomo si costituisce anche grazie alla capacità d'incontro con l'altro da sé, anzi la storia stessa è resa possibile dall'esercizio della libertà nella dialogicità costitutiva dell'esistenza che, tuttavia, conserva una stupefacente transtoricità nei soggetti umani.

Proseguendo nell'analisi, osserva che l'immagine della realtà, che scaturisce da un incontro storico, entra in relazione, in modo riflesso e dunque mediato, con l'immagine stessa dell'io dell'uomo e contribuisce alla sua conformazione. Ciò si rivela un evento non trascurabile in quanto l'uomo, sino a che tale immagine è ritenuta veritiera, vive conformemente a essa. Jonas così argomenta:

\footnotetext{
${ }^{5}$ ID., Überleitung. Von der Philosophie des Organismus zur Philosophie des Menschen, in ID., Organismus und Freiheit, cit, p. 263; trad. it., Transizione dalla filosofia dell'organismo alla filosofia dell'uomo, in
} Organismo e libertà, cit., p. 237. 
L'uomo in senso pieno compare, quando egli, che ha dipinto il toro e persino il suo cacciatore, si volge a gettare lo sguardo sull'immagine, che non può venire dipinta, del proprio comportamento e stato d'animo. Attraverso la distanza di questo sguardo di meraviglia, ricerca e paragone si costituisce il nuovo essere "io". Questa è fra tutte la più grande avventura della mediatezza e dell'oggettivazione ${ }^{6}$.

La parola "immagine" è qui utilizzata per porre in evidenza che l'uomo modella, esperisce e giudica il proprio essere interno e il proprio agire esterno secondo l'immagine di ciò che si addice a un essere umano. Consciamente o inconsciamente egli opera le sue scelte in base all'idea di uomo che ha introiettato in accordo o in conflitto, riconoscendola o negandola. Tale immagine-guida non viene mai meno anche se subisce dei mutamenti, delle trasformazioni nel corso della vita, è elaborata e conservata nelle varie forme comunicative della società e l'individuo la riceve già articolata. Scrive Jonas:

\begin{abstract}
Come impara da altri a vedere e discutere cose, così da queste impara a vedere se stesso e a esprimere ciò che vede lì "a immagine e somiglianza" del modello sussistente. Ma imparando ciò, imparando a dire "io", egli scopre potenzialmente anche la propria identità nella sua solitaria unicità. Una privata oggettività del sé è pertanto in costante rapporto con l'immagine pubblica dell'uomo e contribuisce attraverso la propria esteriorizzazione alla continua trasformazione di essa: il contributo anonimo di ogni sé alla storia di tutti ${ }^{7}$.
\end{abstract}

Se l'individuo si adegua completamente a ciò che riceve si fa assorbire dal modello generale, oppure può ritirarsi nella propria solitudine o ancora può agire in modo da contribuire in vario modo al cambiamento del modello dominante.

L'immagine dell'uomo è, da un lato, il prodotto storico della riflessione culturale di un'epoca pur nella dimensione dell'originalità individuale e, dall'altro, non

\footnotetext{
${ }^{6}$ ID., Überleitung. Von der Philosophie des Organismus zur Philosophie des Menschen, in ID., Organismus und Freiheit, cit, p. 261; trad. it., Transizione dalla filosofia dell'organismo alla filosofia dell'uomo, in Organismo e libertà, cit., p. 235.

${ }^{7}$ Ivi, p. 261; trad. it. cit., p. 236. 
potendo prescindere da elementi naturali e culturali che permangono nella caratterizzazione degli esseri umani, presenta elementi metastorici.

\section{Quaestio mihi factus sum}

Nel mondo che esperiamo possiamo osservare che l'evoluzione viene continuata dalla storia umana e la biologia fa spazio alle scienze umane, alla filosofia e alla religione.

Nelle religioni del Libro, ad esempio, gli elementi metastorici, vengono messi in un rapporto di derivazione dall'immagine stessa di Dio. Gli elementi transtorici presenti nell'umanità di tutti i tempi possono essere espressi per i credenti sinteticamente nella considerazione che l'essere creati a immagine di Dio, significa vivere con un'immagine dell'uomo.

Il sorgere e lo svilupparsi dei fattori culturali accanto a quelli naturali avvengono proprio a partire dall'emergere e dal perfezionarsi nella scala evolutiva della capacità di produzione di immagini. Le rappresentazioni, nello spazio autonomo creatosi nella distanziazione e mediatezza rispetto all'esperienza dell'immediatezza dell'incontro diretto con l'oggetto, danno all'essere vivente la possibilità della riflessione fino a giungere nell'essere umano alla possibilità dell'autoriflessione. Così nascono e si sviluppano religione, etica e metafisica, per fornire risposte all'essere umano che è diventato domanda a se stesso: "Quaestio mihi factus sum... nell'orizzonte di un'interpretazione dell'intero essere ${ }^{8}$.

Questa originaria domanda, sorta inevitabilmente nella possibilità di riflettere sull'esperienza della morte, testimoniata già agli albori della storia dalla memoria delle vite passate racchiusa nelle tombe, tipico artefatto del genere umano, si è declinata in vari modi nel corso del tempo.

\footnotetext{
8 H. JONAS, Überleitung. Von der Philosophie des Organismus zur Philosophie des Menschen, in ID., Organismus und Freiheit, cit., p. 262; trad. it., Transizione dalla filosofia dell'organismo alla filosofia dell'uomo, in Organismo e libertà, cit., p. 237. 
Hans Jonas dichiara ai propri studenti al termine del corso del 1966 e successivamente in quello del 1970, tenuti entrambi alla New School for Social Research, Graduate University of New York, che «l'atteggiamento del filosofo deve essere quello di non credere ${ }^{9}{ }^{9}$, poiché credere è l'atteggiamento dell'uomo in quanto ebreo, cristiano, musulmano ecc.. Il filosofo indaga le questioni a cui si trova di fronte a partire dall'esperienza con gli strumenti della ragione, intesa in senso lato antico e partecipativo, la quale si presenta, infatti, ancora in qualche modo comune agli umani se utilizzata nell'attenzione continua alla falsificazione che l'oggetto può operare sulle costruzioni del soggetto. In tale prospettiva egli cerca di rispondere, in primo luogo da filosofo, all'originaria Quaestio mihi factus sum, descrivendo la posizione dell'essere umano nell'essere del mondo, nel cosmo, e individuandone conseguentemente le caratteristiche precipue e il ruolo, che possono essere espressi rappresentativamente nel binomio: libertà e responsabilità.

La filosofia di questo autore, a noi contemporaneo, muove dalle primitive e radicali domande che ogni uomo, dopo la constatazione dell'esistere, pone a se stesso per orientarsi nel mondo: «Dove sono? Che devo fare?». A tali domande Jonas risponde per mezzo degli strumenti e dei contenuti conoscitivi del filosofo, dell'uomo di cultura della sua epoca, con la ricerca e la descrizione di un'ontologia che renda conto della necessità e della libertà, che esperiamo continuamente, e con la teoria della responsabilità dell'essere umano, che consegue appunto alla sua possibilità di libertà.

II senso di questo dover fare responsabile dell'uomo viene giustificato razionalmente ed empaticamente nella prospettiva relazionale-partecipativa dell'essere umano all'essere del mondo e comporta il non potersi esimere anche dalla

\footnotetext{
9 ID., Problems of Freedom '66, in A. Michelis, Libertà e responsabilità nella filosofia di Hans Jonas del secondo dopoguerra, vol. II, Appendice, Università degli Studi di Torino, Dottorato di ricerca in Filosofia ed Ermeneutica filosofica, ciclo XVII, a.a.2001-2005, p. 653 e p. 456 (testo inglese e trad. it. dell'inedito dattiloscritto del corso di H. Jonas tenuto alla New School for Social Research of New York nel 1966); ID., Problems of Freedom '70, in ID., Problemi di Libertà, intr. e cura di E. Spinelli, trad. it. e postfaz. di A. Michelis, Torino, Nino Aragno Editore, 2010, p. 409 originaleinglese e p. 198 trad. it. del corso del 1970, che è stato pubblicato qui per la prima volta.
} 
cura della continuazione, come è reso archetipicamente evidente nel rapporto di filiazione.

Si possono certamente rintracciate cause, ragioni per cui c'è il male nel mondo, inteso qui come sofferenza, bisogno, mancanza, finitezza. Tale male è conosciuto dall'individuo come l'ostacolo alla propria realizzazione o a quella di coloro i quali egli si cura; è conosciuto come la propria distruzione o quella degli individui della sua specie o di una parte della natura. II tutto, però, riesce ad assumere un significato più accettabile, anche razionalmente, se visto in un prospettiva più ampia che tenga conto delle parti e dell'insieme in divenire ${ }^{10}$.

Queste riflessioni avvengono in una dimensione del pensare che Jonas stesso connota come "orizzontale» e dunque storico. In un saggio intitolato Immortalità ed esistenza odierna, posto a conclusione di Organismo e libertà, egli sostiene che la filosofia contemporanea non possa più pensare il tempo soltanto come forma fenomenica di una realtà atemporale.

Dalla scoperta della fondamentale storicità dell'uomo sino all'elaborazione ontologica della più intima temporalità del suo essere siamo stati resi consapevoli del fatto che il tempo, ben lungi dall'essere mera forma di apparenze, appartiene piuttosto all'essenza di tale essere come quella del sé e che per ogni singolo séla sua finitezza èla condizione indispensabile della possibile autenticità del suo esistere ${ }^{11}$.

${ }^{10}$ Cfr. H. JONAS., The Burden and Blessing of Mortality, in ID., Mortality and Morality. A Search for the Good after Auschwitz, cit., pp. 87-98; trad. it. di A. Benussi, Peso e benedizione della mortalità, in Tecnica, medicina ed etica. Prassi del principio responsabilità, a cura e intr. di P. Becchi, Torino, Einaudi, 1997, pp. 206-221. Cfr. inoltre ID., Session IV, in ID., Problems of Freedom '66, cit., pp. 520-537 e pp. 327-343; ID., Lesson IV, in ID., Problemi di Libertà, cit., pp. 295-309 e pp. 55-73.

11 ID., Unsterblichkeit und heutige Existenz, in ID., Organismus und Freiheit, cit., p. 323; trad. it., Immortalità ed esistenza odierna, in Organismo e libertà, cit., p. 290. Cfr. ID., Zwischen Nichts und Ewigkeit. Zur Lehre vom Menschen, - Anhang: Aus einem Briefwechsel zwischen Rudolf Bultmann und dem Verfasser anlässlich des Aufsatzes über die Unsterblichkeit, Göttingen, Vandenhoeck \& Ruprecht, 1987, pp. 63-72; trad. it. di M. Sinatra - F. Aster, Tra il nulla e l'eternità., - Appendice. Da un carteggio tra Rudolf Bultmann e l'Autore in occasione del saggio sull'immortalità, a cura di G.R. Rilke, Ravenna, Gallio, 1992, pp. 96-109. 
Per gli uomini contemporanei permeati di cultura occidentale è mentalità comune che il qui non possa e non debba essere scambiato con un là ${ }^{12}$. Anche nell'accettazione più razionale e nell'interiorizzazione più empatica del nostro destino di mortali, che tutti accomuna, con tutto il peso e la benedizione che esso può comportare, rimane tuttavia enigmatico, da un lato, il risorgere continuo del desiderio insopprimibile che la storicità, con il suo mondo dialetticamente conflittuale e per lo più ingiusto agli occhi umani, non sia l'ultima verità, e, da un altro lato, il permanere intatto nei secoli del mistero dell'origine dell'universo.

\section{Il problema del senso}

La filosofia, le scienze dell'uomo e della natura lasciano insolute questioni fondamentali per la chiarificazione ultima di quelle domande che esse stesse si pongono e con cui paiono destreggiarsi con sufficiente efficacia nello sviluppare le questioni su un piano orizzontale e contestuale. Jonas, a questo proposito, fenomenologicamente si chiede se l'eternità possa avere una relazione con il temporale, per quanto breve e rara sia potrebbe servirci da indice di una partecipazione del nostro essere o sostanza all'eternità, ovvero di un'immortalità.

In quali situazioni e in quale forme incontriamo l'eterno? Quand'è che sentiamo le ali dell'eterno sfiorare il nostro cuore e rapire al tempo l'ora? Come entra l'assoluto nella relatività del nostro essere quotidiano? ${ }^{13}$

Egli per cercare una risposta a tali domande non si richiama alle esperienze mistiche, che peraltro non conosce, ma la cui testimonianza sarebbe comunque decostruita dalla psicoanalisi e dalla psicologia contemporanee. Nemmeno cita gli incontri elitari con l'amore e la bellezza, in cui si intravede uno scorcio di eternità, ma che possono provocare l'umiliazione dei molti a cui sono negati. Come un uomo del suo tempo, invece, cerca quel tipo di evidenza che può ritrovare nell'attività del

${ }^{12}$ Cfr. ID., Unsterblichkeit und heutige Existenz, in ID., Organismus und Freiheit, cit., pp. 322; trad. it., Immortalità ed esistenza odierna, in Organismo e libertà, cit., p. 289.

${ }^{13}$ Ivi, p. 323; trad. it. cit., pp. 291. 
soggetto e la individua negli attimi della decisione, quando tutto l'essere si impegna e sente di agire in una prospettiva di eternità, come davanti agli occhi di Dio. Per esprimere quel sentimento forte ed evidente deve, comunque, ammettere di dover ricorrere ai simboli della fede religiosa o a immagini vicine ${ }^{14}$. Ad esempio, afferma Jonas, in mancanza della certezza della fede, può intervenire l'angoscia che ci giunge dall'immagine del rischio ultimo e totale di fronte ai limiti estremi dell'esistenza, agli ultimi attimi concessi dal tempo.

\footnotetext{
Agire come di fronte alla fine è agire come di fronte all'eternità, lo è cioè quando sia l'una cosa chel'al tra vengono comprese come appello alla verità in sé. [...] comprendere la fine in questo modo significa proprio comprenderla in una luce al di là del tempo ${ }^{15}$.
}

Accade così il paradosso di cercare, proprio in una situazione auto-negante rispetto alla durata, l'indefinita relazione con l'eternità; anzi è tale paradosso che può extra-concettualmente lasciar trapelare qualcosa su quel sentimento di eternità che troviamo in noi. Il punto dell'attimo e non l'estensione del flusso è il nostro contatto con l'eternità. Jonas intende però non l'attimo passivo nunc stans, l'ora stante, del mistico, ma l'attimo che tiene attivamente sospeso il tempo sulla soglia dell'azione come nell'atemporale.

\footnotetext{
Presto inghiottito dal movimento che ha messo in moto, l'attimo segna la nostra apertura verso la trascendenza, proprio perché ci affida al fugace della situazione, e in questa doppia esposizione, in cui consiste l'essenza dell'interesse incondizionato, pone colui che agisce in modo responsabile fra eternità e tempo ${ }^{16}$.
}

L'essenza della storicità dell'essere umano emerge in questa «terra di mezzo» dove sempre si attende la possibilità di un nuovo inizio, qui e ora. Nel saggio intitolato

\footnotetext{
${ }^{14}$ Cfr. ivi, pp. 323-324; trad. it. cit., p. 291.

${ }^{15}$ Ivi, p. 325; trad. it. cit., p. 292.

${ }^{16}$ Ivi, p. 326; trad. it. cit., p. 293.
} 
Mutamento e permanenza: sulla possibilità di comprendere la storia ${ }^{17}$, per Jonas è la possibilità della storia che rimanda al metastorico, nei suoi elementi invarianti. Di tutto ciò che si pone oltre la storia, oltre l'esperienza del costante divenire del tutto che si dà ai sensi e alla ragione, se ne può parlare, però, solo dilatando i concetti, le categorizzazioni dell'esperienza, tramite il sentimento del desiderio in immagini metaforiche o simboliche quali troviamo nel mito $^{18}$ e nelle religioni. Per Jonas il mito è la proiezione della realtà esistenziale in una costruzione narrativa che riesca a dare un senso al mondo nel suo insieme.

Di tal genere è il mito ipotetico che voglio credere "vero", nel senso che attraverso una buona dose di fortuna un mito può adombrare una verità necessariamente inconoscibile e addirittura ineffabile in concetti diretti, ma che tuttavia, attraverso automanifestazioni nella nostra più profonda esperienza, avanza pretese verso la nostra capacità di renderne indirettamente conto in immagini revocabili e antropomorfiche. ${ }^{19}$

Questo mito ipotetico è quello della creazione del mondo da parte di Dio, o mente creatrice $^{20}$, che viene all'idea, quando l'Autore lascia agire «la libertà dell'ignoranza» nell'invenzione plausibile. Eccone uno stralcio:

Perché il mondo sia e sia per se stesso, Dio rinunciò al proprio essere; si spogliò della sua divinità per riceverla indietro dalla sua odissea del tempo, carica del raccolto casuale dell'imprevedibile esperienza temporale, trasfigurata o forse anche sfigurata da essa. In una tale auto-rinuncia dell'integrità divina per il divenire incondizionato non si può concedere nessun al tro sapere preliminareal di là di quello riguardo alle possibilità che

17 Cfr. ID., Change and Permanence: On the Possibility of Understanding History, in ID., Philosophical Essays. From Ancient Creed to Technological Man, Englewood Cliffs (NJ), Prentice-Hall, 1974, pp. 237 260; trad. it di G. Bettini, Mutamento e permanenza: sulla possibilità di comprendere la storia, in Dalla fede antica all'uomo tecnologico. Saggi filosofici, a cura e intr. di A. Dal Lago, Bologna, il Mulino, 1991, pp. 343-371.

${ }^{18}$ Cfr. H. JONAS, Myth and Mysticism: A Study of Objectification and Interiorization in Religious Thought, in ID., Philosophical Essays. From Ancient Creed to Technological Man, cit., p. 291; trad. it., Mito e misticismo. Un'analisi dell'oggettivazione e dell'interiorizzazione del pensiero religioso, in Dalla fede antica all'uomo tecnologico. Saggi filosofici, cit., p. 411.

19 ID., Unsterblichkeit und heutige Existenz, in ID., Organismus und Freiheit, cit., pp. 335; trad. it., Immortalità ed esistenza odierna, in Organismo e libertà, cit., p. 301.

${ }^{20}$ Cfr. H. JONAS, Matter, Mind, and Creation, in ID., Mortality and Morality. A Search for the Good after Auschwitz, cit., pp. 180 e 183 e ss. 
I'essere cosmico concede attraverso le proprie condizioni: proprio a queste condizioni Dio affidò la sua causa, spogliandosi a favore del mondo. ${ }^{21}$

Rimane forse una pregnante memoria di ciò nel ruotare della materia e si forma l'attesa presaga con cui l'eterno accompagna sempre più l'evoluzione nel tempo quale un paziente emergere della trascendenza dall'opacità dell'immanenza. II mistero dell'origine del mondo ${ }^{22}$, permane intatto anche con il progredire iperbolico del nostro sapere scientifico, culturale, del nostro potere tecnologico e alla domanda "perché c'è qualcosa piuttosto che il nulla" segue il silenzio della razionalità moderna e contemporanea. Così il mito religioso della creazione, comune a più religioni, anche se in declinazioni e simbologie differenti, dà una risposta immaginifica e in qualche modo pacificante, pur non sopprimendo la dimensione del rischio intrinseco all'esistenza, a un desiderio primordiale di conoscere l'origine del dato di fatto dell'esistenza, che rimane altrimenti «il mistero dei misteri». II mito nella versione cara a Jonas, racconta che l'eterno fondamento permise al mondo di essere, rinunciando alla propria inviolabilità ${ }^{23}$. L'autonegazione, che si trasforma in spazio di libertà, è la prima causa delle possibilità dell'esistenza di ogni creatura.

In tale visione mitologico-religiosa, avviene una sorta di contrazione del potere di $\mathrm{Dio}^{24}$ e un ampliamento del potere umano. Ne risulta che il corso del creato non è più né stabilito né garantito da Dio solo e l'uomo può incidere, pur nei limiti di un essere finito e mortale, nella realizzazione del bene e del male, inteso in senso fisico come sofferenza, ma anche in senso morale.

\footnotetext{
${ }^{21}$ H. JONAS, Unsterblichkeit und heutige Existenz, in ID., Organismus und Freiheit, cit., p. 332; trad. it., Immortalità ed esistenza odierna, in Organismo e libertà, cit., p. 298.

${ }^{22}$ Cfr. ID., La domanda senza risposta. Alcune riflessioni su scienza, ateismo e la nozione di Dio, trad. it., cura e intr. di E. Spinelli, Genova, II Melangolo, 2001 (pubblicazione di inedito con testo ingl. a fronte).

${ }^{23}$ Cfr. ID., Matter, Mind, and Creation, in ID., Mortality and Morality. A Search for the Good after Auschwitz, cit., pp. 189-191. II paragrafo è intitolato: Alternative Speculation of Cosmogony: God's Renunciation of Power in Favor of Cosmic Autonomy and Its Chances.

${ }^{24}$ Cfr. M. GIULIANI, Hans Jonas. Un concetto di Dio non (più) onnipotente, in ID., Auschwitz nel pensiero ebraico. Frammenti dalle "teologie dell'Olocausto", Brescia, Morcelliana, 1998, pp. 111-115. 
La riflessione mitologico-religiosa di Jonas, che nasce dalle domande di un uomo credente a fronte della crudezza della storia ${ }^{25}$, giunge alla massima considerazione possibile della libertà e della responsabilità dell'essere umano nel mondo. Si conclude, dunque, sotto il segno della speranza e della fiducia, che gli eventi non hanno mai totalmente soffocato, nelle capacità umane e nella positività di essere uomini e donne, creati a immagine di Dio. Tale fiducia era già sostanzialmente presente nelle sue riflessioni teologiche sul cristianesimo ${ }^{26}$, iniziate alla scuola di Martin Heidegger e Rudolf Bultmann ${ }^{27}$ nel periodo degli studi universitari in Germania; ed è anche la chiave interpretativa della sua simpatia per Pelagio nella disputa con Agostino sulla questione del rapporto fra libertà umana e grazia divina, propensione resa evidente nelle lezioni finali di Problems of Freedom del 1966 e del $1970^{28}$.

\section{Il filo rosso}

La libertà e la responsabilità dell'uomo, la fiducia della loro possibilità, sono comunque il filo rosso di tutta la riflessione e il lavoro di ricerca jonasiano, accompagnato da durissime esperienze personali ed epocali.

${ }^{25}$ Cfr. H. JONAS, Der Gottesbegriff nach Auschwitz. Eine jüdische Stimme, Frankfurt a/M, Suhrkamp, 1987; trad. it e intr. di C. Angelino, II concetto di Dio dopo Auschwitz. Una voce ebraica, Genova, il melangolo, 1993.

${ }^{26}$ Cfr. H. JONAS, Augustin und das paulinische Freiheitsproblem. Ein philosophischer Beitrag zur Genesis der christlich-abendländischen Freiheitsidee, Göttingen, Vandenhoeck \& Ruprecht, 1930 (1965 seconda ed. integrata, intr. di J.M. Robinson); trad. it. dell'ed. 1965 a cura di C. Bonaldi, Agostino e il problema paolino della libertà. Studio filosofico sulla disputa pelagiana, intr. di C. Bonaldi e postfaz. di A. Michelis, Brescia, Morcelliana, 2007.

${ }^{27}$ Cfr. F. BIANCO, Hans Jonas tra Heidegger e Bultmann, in «Paradigmi », 22 (2004), n. 66, pp. 303-317; F. CIARAMELLI, Sans abri. Phénoménologie de la liberté et question du mal chez Hans Jonas, in "Études Phénoménologiques ", 17 (2001), n. 33-34, pp. 131-154.

${ }^{28}$ Cfr. H. JONAS, Problems of Freedom '66, cit., Session 9 e ss; ID. Problemi di Libertà, cit., Lesson 9 e ss. Inoltre, ID., The Abyss of the Will: Philosophical Meditation on the Seventh Chapter of Paul's Epistle to the Romans, in ID., Philosphical Essays. From Ancient Creed to Technological Man, cit., pp. 335-348; trad. it., L'abisso della volontà. Meditazioni filosofiche sul settimo capitolo della lettera ai Romani di Paolo, in Dalla fede antica all'uomo tecnologico. Saggi filosofici, cit., pp. 465-481. 
Tornando alla riflessione filosofica, campo d'indagine della nostra analisi, abbiamo visto come egli individui descrittivamente e concettualmente ${ }^{29}$ nella libertà un carattere ontologico fondamentale della vita nel suo sviluppo evolutivo. Ogni essere vivente, che esiste nel tempo, è per necessità materialmente concreto e non può continuare a esistere in una separazione dalla sua sostanza materiale, ma ciò, per il nostro autore, non significa assoluta e precisa coincidenza con la determinata somma di materia che lo compone chimicamente.

La possibilità di trascendere la pura fissità meccanica della materia è evidente nel rinnovamento cellulare della vita organica, dove accade una continua e complessa trasformazione non del tutto determinabile in modo predittivo. Tale possibilità risulta, comunque, anche presente nella procedura della scelta in situazioni in cui non si può risalire a una causalità lineare e incontrovertibile. L'“azione che sceglie” non è propria soltanto degli esseri umani, ma a livello istintivo ed emotivo si può osservare anche tra gli animali; e nella indeterminatezza della previsione di alcune combinazioni chimiche, senza la descrizione storica del contesto, è una possibilità innegabilmente presente in biologia fin dalle primitive forme di vita.

Pur ammettendo con mentalità scientifico-razionale che ciò che permette il passaggio dalla sostanza inanimata a quella vivente deve essere una tendenza insita nell'essere stesso, se sempre vale il principio della fisica classica newtoniana, che nel mondo che esperiamo, nulla si crea e nulla si distrugge, ma tutto si trasforma, ci troviamo, comunque, nella filosofia di Jonas, a un restringimento di campo per quanto riguarda l'applicazione del principio interpretativo della causali tà lineare necessitante.

Jonas dà nuovo spazio al principio della libertà, come possibilità effettiva di scontrarsi contro i limiti dati e di elevarsi oltre i meccanismi della materialità, oltre la pura necessità. La novità della sua proposta consiste nel non sclerotizzare in una contrapposizione schematica e sterile questi principi interpretativi del mondo, la causalità e la libertà, che colgono aspetti della realtà potenzialmente contrapposti e

${ }^{29}$ Cfr. anche ID., Ist Gott ein Mathematiker? Vom Sinn des Stoffwechsels, in ID., Organismus und Freiheit, cit., pp. 130-131; trad. it., Dio è un matematico?, in Organismo e libertà, cit., pp. 116-117. 
contraddittori. Egli ricerca una loro composizione per poter meglio leggere la complessità del reale, che manifesta proprio nell'esperienza anche quotidiana, di cui ogni essere umano può essere testimone, aspetti di causalità necessitante e aspetti di libertà.

Mantenendo la prospettiva evoluzionistica nei suoi tratti sostanziali, Jonas rende evidente come anche nella selezione e nelle mutazioni che avvengono nel passaggio fra i gruppi dei viventi, quali ad esempio i vegetali e gli animali, vi siano degli elementi di innovazione e di sovrabbondanza degli effetti, che risultano irrintracciabili in una sequenza di cause necessitanti, senza far intervenire principi di indeterminatezza. Nell'uomo emerge poi una sorprendente sovrabbondanza di caratteristiche in qualche modo permanenti oltre a ciò che è necessario per la sua sopravvivenza, come l'autonomia, il poter autogenerare dei fini e l'ipersviluppo di alcune capacità d'astrazione. Tale sovrabbondanza, proprio se osservata con l'onestà e l'umiltà tipica dell'uomo di scienza, che ricerca la verità come possibilità di riscontro di un'oggettualità nell'esperienza, non può non mettere in crisi quelle teorie interpretative che riducono il fenomeno della vita e il mondo al meccanicismo assolutamente deterministico di una pura materia.

Il pensiero di Jonas, in particolare quello della seconda fase, dedicato alla filosofia della biologia, nel confronto filosofico con la scienza crea nuovi spazi di riflessione critica per la filosofia e per le scienze stesse; in tale incontro queste acquisiscono maggior coscienza metodologica e ampliano le prospettive di osservazione dei fenomeni.

Sorgono, così, soluzioni interpretative che, attraversando e superando desueti schieramenti ideologici, aprono a nuove possibilità di percorsi al fine di riuscire a comprendere più in profondità la complessità della realtà epocale a cui ci si trova di fronte e di aumentare la capacità di interagire con essa. 


\section{Referenze}

H. JONAS, Augustin und das paulinische Freiheitsproblem. Ein philosophischer Beitrag zur Genesis der christlich-abendländischen Freiheitsidee. Göttingen, Vandenhoeck \& Ruprecht: 1930 (1965 seconda ed. integrata, intr. di J.M. Robinson); trad. it. dell'ed. 1965 a cura di C. Bonaldi, “Agostino e il problema paolino della libertà. Studio filosofico sulla disputa pelagiana", intr. di C. Bonaldi e postfaz. di A. Michelis, Brescia, Morcelliana, 2007.

H. JONAS, Zwischen Nichts und Ewigkeit. Zur Lehre vom Menschen. Göttingen, Vandenhoeck \& Ruprecht, 1963 (1987); trad. it. di M. Sinatra - F. Aster, "Tra il nulla e l'eternità“, a cura di G.R. Rilke, Ravenna, Gallio, 1992.

H. JONAS, Organismus und Freiheit. Ansätze zu einer philosophischen Biologie. Göttingen, Vandenhoeck \& Ruprecht, 1973; trad. it. di A. Patrucco Becchi, in "Organismo e libertà. Verso una biologia filosofica", a cura e intr. di P. Becchi, Torino, Einaudi, 1999.

H. JONAS, Philosophical Essays. From Ancient Creed to Technological Man. Englewood Cliffs (NJ), Prentice-Hall, 1974; trad. it di G. Bettini, "Dalla fede antica all'uomo tecnologico. Saggi filosofici", a cura e intr. di A. Dal Lago, Bologna, il Mulino, 1991.

H. JONAS, Das Prinzip Verantwortung. Versuch einer Ethik für die technologische Zivilisation. Frankfurt a/M, Insel Verlag, 1979; trad. it. di P. Rinaudo, "Il principio responsabilità. Un'etica per la civiltà tecnologica", a cura e intr. di P.P. Portinaro, Torino, Einaudi, 1990.

H. JONAS, Der Gottesbegriff nach Auschwitz. Eine jüdische Stimme. Frankfurt a/M, Suhrkamp, 1987; trad. it e intr. di C. Angelino, "Il concetto di Dio dopo Auschwitz. Una voce ebraica", Genova, il melangolo, 1993.

H. JONAS, Mortality and Morality. A Search for the Good after Auschwitz. L. Voegel (ed., intr.), Evanston (Illinois), Northwestern University Press, 1996. 
H. JONAS, La domanda senza risposta. Alcune riflessioni su scienza, ateismo e la nozione di Dio, trad. it., cura e intr. di E. Spinelli, Genova, II Melangolo, 2001 (pubblicazione di inedito con testo orig. ingl. a fronte).

H. JONAS, Problems of Freedom 66, in A. Michelis. Libertà e responsabilità nella filosofia di Hans Jonas del secondo dopoguerra. Vol. II, "Appendice", Università degli Studi di Torino, Dottorato di ricerca in Filosofia ed Ermeneutica filosofica, ciclo XVII, a.a.2001-2005 (testo inglese e trad. it. dell'inedito dattiloscritto del corso di H. Jonas tenuto alla New School for Social Research of New York nel 1966).

H. JONAS, Problems of Freedom 70, in ID., Problemi di Libertà, intr. e cura di E. Spinelli, trad. it. e postfaz. di A. Michelis, Torino, Nino Aragno Editore, 2010 (pubblicazione di inedito con testo originale ingl.).

M. GIULIANI, Hans Jonas. Un concetto di Dio non (più) onnipotente, in ID., Auschwitz nel pensiero ebraico. Frammenti dalle 'teologie dell'Olocausto. Brescia, Morcelliana, 1998, pp. 111-115.

F. CIARAMELLI, Sans abri. Phénoménologie de la liberté et question du mal chez Hans Jonas, in «Études Phénoménologiques», 17 (2001), n. 33-34, pp. 131-154.

F. BIANCO, Hans Jonas tra Heidegger e Bultmann, in «Paradigmi», 22 (2004), n. 66, pp. 303-317.

Doutora em Filosofia pela Universidade deTorino (Itália) E-mail:angmich@tin.it 